\title{
Zweites Kapitel \\ Die Theorie des logischen Symbolismus als Hauptthema des Tractatus
}

Wenn man versucht, die Sätze des Tractatus zu überblicken und im Netz seiner Begriffe die Themen zu bestimmen, die als Gegenstand von Theorien mit mehr oder weniger festgelegten Bezeichnungen erfasst werden können, kommt vor allem die folgende Zuordnung von Sätzen und Theorien in Betracht. Die Sätze 1, 2 und 3, die sich mit der Charakterisierung des Gedankens als logischen Bildes durch seine Beziehungen zu dem von ihm Abgebildeten, zu seinem Ausdruck (dem Satz) und den Bestandteilen desselben beschäftigen, sowie der Satz 4, der den sinnvollen Satz als ein logisches Bild definiert, sind Ausdruck der Bildtheorie des Tractatus. Den Satz 4 zusammen mit dem Satz 5, der die Beziehungen des sinnvollen Satzes zu anderen Sätzen charakterisiert, kann man als Formulierung der Theorie der Wahrheitsfunktionen betrachten ${ }^{1}$. Einige Formulierungen der Sätze 4 und 5, der Satz 6, welcher die Regel der Konstruktion der Sätze formuliert und den Funktionen der logischen Sätze gewidmet ist, sowie der Satz 7 bilden den Bestand der Theorie des Zeigens ${ }^{2}$. Diese Zuordnung kann nicht eine strenge thematische Abgrenzung der Sätze des Tractatus einerseits und der Theorien andererseits beanspruchen, sofern Begriffe, die als Grundbegriffe

\footnotetext{
${ }^{1}$ Die Theorie der Wahrheitsfunktionen wird manchmal nicht nur als Ergänzung zur Bildtheorie, sondern sogar als ihre Facette gedeutet, wodurch die Bildtheorie mit einer Bildauffassung der Wahrheit gleichgesetzt wird. S. M.B. Hintikka, J. Hintikka. Untersuchungen zu Wittgenstein. Frankfurt am Main, Suhrkamp, 1996, 141, 151, J. Hintikka, ,'Die Wende der Philosophie': Wittgensteins New Logic of 1928“ (im weiteren: Hintikka.Wende). In: S. Teghrarian, A. Serafini (Hrsg.). Wittgenstein and Contemporary Philosophy. Wakefield, New Hampshire, Longwood Academic, 1992, 1-35, 6. ${ }^{2}$ Diese Bezeichnung (die Bezeichnung als ,,doctrine of showing“) übernehme ich von Griffin. S. J. Griffin. Wittgenstein's Logical Atomism. Seattle, London, University of Washington Press, 1969, 18. Diese Theorie wird auch als Theorie des Sagens und Zeigens charakterisiert, z.B. von Geach. S. P.T. Geach. „Saying and Showing in Frege and Wittgenstein“. In: Acta Philosophica Fennica, vol. XXVIII, 1976. Essays on Wittgenstein in Honour of G.H. von Wright. 54-70, 55.
} 
einer der Theorien auftreten, auch in dem Aufbau anderer Theorien mit einbegriffen sind.

Die ersten fünf Sätze des Tractatus kann man, sowohl von dieser Zuordnung als auch von anderen Auffassungsprinzipien ausgehend, als Formulierung der Thesen charakterisieren, welchen der Begriff des Sinnes zugrunde liegt. Der Grundbegriff der Gesamtheit von Thesen, die in den Sätzen 6 und 7 dargelegt sind, ist dann der Begriff des Sinnlosen. Eine solche Auffassung wird von Autoren vertreten, die diese zwei Begriffe zusammen mit dem Begriff des Unsinns als zentrale Kategorien des Tractatus behandeln ${ }^{3}$. Die Wurzeln dieser Behandlungsweise sehe ich in dem Versuch, einige Thesen Wittgensteins auf die Analyse des Textes des Tractatus anzuwenden. Dieser Versuch wurzelt im Satz 6.54 des Tractatus sowie in verschiedenen Auslegungen dieses Satzes und schlägt oft, nach einer treffenden Bemerkung von Wrights, in ein Missverstehen um, das manchmal sogar ins Verspotten ausartet ${ }^{4}$. Dadurch, dass die Begriffe des Sinnes und des Unsinns in Mittelpunkt der Rekonstruktion des Tractatus gerückt werden, will man auch einem solchen Missverstehen trotzen.

Sofern der Sinn ein Charakteristikum des Satzes ist, gehe ich von dem sekundären Charakter dieser letzteren Auffassung des Tractatus aus und möchte zeigen, dass die oben erwähnten drei Theorien - die Bildtheorie, die Theorie der Wahrheitsfunktionen und die Theorie des Zeigens - die Komponenten einer sie umfassenden Theorie sind. Diese bezeichne ich als Theorie des logischen Symbolismus. Als ihren Grundbegriff betrachte ich den Begriff eines logischen Symbols.

\footnotetext{
${ }^{3}$ So sind laut Friedlander Sätze 6 und 7 des Tractatus nicht nur dem Begriff des Sinnlosen, sondern auch dem Begriff des Unsinns, der genau wie Sinn zur Sprache gehört, gewidmet: Der Unsinn entsteht an den Grenzen der Sprache bei dem Versuch, diese Grenzen und somit die Bedingungen der Sprache zu überwinden, und ist als Erfahrung der Welt bedeutungsvoll oder signifikant. E. Friedlander. Signs of Sense. Reading Wittgenstein's Tractatus (im weiteren: Friedlander). Cambridge, Massachusetts, London, England, Harvard University Press, 2001, 22-23, 25, 116-121, 147, 151-153, 199 , 205. S. auch M. Kremer. Book Review of: Ian Proops, Logic and Language in Wittgenstein's Tractatus. In: The Philosophical Review, Vol. 111, No. 2, 2002, 327-330, 328.

${ }^{4}$ G.H. von Wright. "Modal Logic and the Tractatus" (im weiteren: von Wright.Modal Logic). In: G.H. von Wright. Wittgenstein. Oxford, Basil Blackwell, 1982, 183-200, 186
} 
Die erste Frage, die sich im Zusammenhang mit meiner Betrachtungsweise erhebt, ist folgende. Warum will man die Theorie des Tractatus als Theorie des logischen Symbolismus bezeichnen, wo es im Tractatus nur um Sätze, nämlich um ihren Bestand, die Beziehungen zwischen Sätzen sowie die Relation der Sätze zur Wirklichkeit geht?

Die Antwort auf diese Frage möchte ich auf einem Umweg über einige Thesen der Philosophischen Untersuchungen geben, die es erlauben, den Begriff des Satzes mit dem Begriff der Sprache zu identifizieren. Wittgensteins Analyse seiner früheren Ansichten in den Philosophischen Untersuchungen führt ihn zu der Behauptung, dass die „Frage nach dem Wesen der Sprache, des Satzes, des Denkens ${ }^{\text {c }}{ }^{5}$ der Ausdruck der Vorstellung von der vollkommen zerlegten Form des Ausdrucks (der Sprachform) ist ${ }^{6}$. Diese Vorstellung, die sich im Tractatus in der Behauptung über den gleichen logischen Bau der Sprache und der $\mathrm{Welt}^{7}$ realisiert, ist der Grund dafür, dass laut Wittgenstein die „Begriffe: Satz, Sprache, Denken, Welt, ... in einer Reihe hintereinander [stehen], jeder dem andern äquivalent" ${ }^{\text {"8 }}$. Der Satz und die Sprache werden von Wittgenstein jedoch nicht als Begriffe aufgezählt, die ihre Äquivalenz lediglich einer irrtümlichen Vorstellung verdanken. Obwohl Wittgenstein in den Philosophischen Untersuchungen darauf verzichtet, das, was man „Satz“ und „Sprache“ nennt, als eine formelle Einheit analog dem Tractatus zu behandeln, charakterisiert er es als „Familie mehr oder weniger miteinander verwandter Gebilde“9 oder Sprachspiele. Das Wesentliche der Sprache, d.h. das, was alle Erscheinungsformen der Sprache zur Sprache macht, ist für ihn das Wesentliche des Sprachspiels ${ }^{10}$. Sofern man aber den Begriff davon hat, was man unter „Spiel“" versteht, hat man auch den Begriff davon, was ein Satz ist ${ }^{11}$. Wittgenstein identifiziert darüber hinaus das in der Philosophie der Logik angesiedelte Reden von Sätzen und Worten mit dem Reden vom räumlichen und zeitlichen Phänomen der Sprache ${ }^{12}$. Diese besondere Redeweise ist die

\footnotetext{
${ }^{5} \mathrm{PU}, \S 92$

${ }^{6}$ Ebd., $\$ 91$

${ }^{7}$ TLP, 4.014

${ }^{8} \mathrm{PU}, \S 96$

${ }^{9}$ Ebd., $\$ 108$

${ }^{10}$ Ebd., $\S 65$

${ }^{11}$ Ebd., $\$ 135$

${ }^{12}$ Ebd., $\$ 108$
} 
Voraussetzung dafür, dass eine Theorie des Satzes als Theorie der Sprache betrachtet werden kann.

Wenn man demzufolge den Tractatus als Theorie der Sprache definiert, bleibt noch zu klären, warum die Sprache, um die es im Tractatus geht, ein logischer Symbolismus ist. Zu diesem Zweck möchte ich zunächst den Bestand einer jeden der genannten Theorien durch ihre Grundprinzipien definieren und diese letzteren mit Hilfe des Symbolbegriffs darstellen. Sofern mir dies gelingt, wird dadurch die Möglichkeit bewiesen, eine Theorie des logischen Symbolismus als ein von diesen drei Theorien gebildetes Ganzes zu betrachten. Dass die Theorie des Tractatus als ein solches Ganzes aufgefasst werden kann, möchte ich anschließend durch die Analyse der Bildtheorie begründen.

Den Bestand der Bildtheorie kann man durch folgende Prinzipien definieren:

1. Jedes Bild hat eine Struktur. Für das Symbol Satz, der ein logisches Bild ist, bedeutet das, dass es aus anderen Symbolen (Namen und Sätzen) als seinen strukturellen Elementen besteht und eines strukturellen Zusammenhangs mit anderen Symbolen, nämlich Sätzen, fähig ist $^{13}$.

Während strukturelle Elemente nur in zusammengesetzten Symbolen wie Sätzen aufweisbar sind, können auch einfache Symbole Namen strukturelle Zusammenhänge mit anderen Symbolen, und zwar mit anderen Namen, eingehen, was erlaubt, dieses Prinzip des strukturellen Charakters eines Symbols als eines der Prinzipien der Theorie des logischen Symbolismus zu betrachten.

2. Die Möglichkeit der Struktur, d.h. des Zusammenhangs der Elemente des Bildes, ist die Form der Abbildung. Diese Möglichkeit besteht darin, dass die Dinge sich so zueinander verhalten wie die Elemente des Bildes. Dass das Bild mit der Wirklichkeit die Form der Abbildung gemeinsam hat, ist die Voraussetzung dafür, dass es die Wirklichkeit abbilden kann ${ }^{14}$.

\footnotetext{
${ }^{13}$ TLP, 2.14, 2.141, 2.15, 3.201-3.203, 3.21, 5.2

${ }^{14}$ Ebd., 2.15, 2.151, 2.17. 2.171, 2.2
} 
Für den logischen Symbolismus bedeutet dieses Bedingt-sein des Bildes, dass ein Zeichengebilde gewisse Bedingungen erfüllen muss, um als Symbol zu fungieren: Nicht jedes Zeichen ist ein Symbol.

3. Kraft der Gemeinsamkeit der Form der Abbildung, die das Bild mit der Wirklichkeit teilt, steht es in einer abbildenden Beziehung zur Wirklichkeit. Diese Beziehung besteht in der Zuordnung der Elemente der Struktur des Bildes zu Elementen eines anderen von dem Bild verschiedenen Gebildes ${ }^{15}$. Sofern ein logisches Bild (vor allem Satz) von anderen Bildern dadurch unterschieden wird, dass es Wahrheitsbedingungen hat, ist seine abbildende Beziehung zur Wirklichkeit logisch und wird als darstellend charakterisiert ${ }^{16}$.

Für den logischen Symbolismus bedeutet dieses Prinzip der Zugehörigkeit der abbildenden Beziehung zum Bild, dass die Konstruktion von Symbolen geregelt ist und die Regelung in erster Linie durch Zuordnungen erfolgt.

4. Dass ein Satz wahr oder falsch sein kann, ist sein wesentliches Charakteristikum, das den Satz befähigt, nicht nur das Wissen über die Beziehungen zwischen den Bedeutungen der undefinierbaren Komponenten des Satzes (Namen), sondern mehr Wissen zu vermitteln. So kann man dem Wissen dessen, dass $\mathrm{p}$, das Wissen darüber entnehmen, dass der Satz „p“ wahr und folglich dessen Verneinung falsch ist ${ }^{17}$.

Dank seiner Struktur führt das Symbol somit zu weiteren mit ihm nicht identischen Symbolen hin.

5. Die darstellende Beziehung des logischen Bildes (Satzes) zur Wirklichkeit bestimmt die Stelle des Satzes im Symbolismus (seinen logischen Ort) ${ }^{18}$, sofern sie bestimmt, von welchen anderen Sätzen der Satz unabhängig ist und welche andere Sätze aus ihm folgen oder ihn implizieren.

Der Symbolismus ist mithin ein zusammenhängendes Ganzes.

Die Theorie der Wahrheitsfunktionen beruht auf folgenden Prinzipien:

\footnotetext{
${ }^{15}$ Ebd., 2.1514, 2.032, 2.034

${ }^{16}$ Ebd., 4.462

${ }^{17}$ Notes on Logic, 94

${ }^{18}$ TLP, 3.4, 3.42
} 
1. Jeder Satz ist dann und nur dann ein logisches Bild, wenn er wahr oder falsch ist ${ }^{19}$. Die Bipolarität des Satzes charakterisiert seinen Sinn und unterscheidet den sinnvollen Satz von seinen Bestandteilen Namen: Der Satz beschreibt einen Sachverhalt und benennt ihn nicht $^{20}$. Der Sinn des Satzes ist durch die Angabe seiner Wahrheitsbedingungen definiert ${ }^{21}$ : Laut dem Tractatus besteht die Bestimmung des Sinnes des Satzes in der Bestimmung dessen, unter welchen Umständen der Satz wahr genannt wird ${ }^{22}$.

Bereits 1914 behauptet Wittgenstein, dass Pole zu haben, was dasselbe heißt wie bipolar zu sein, wesentlich für den Satz ist: Dass der Satz sie hat, hat keine Vorbedingung, die sich durch die Forderung ausdrücken lässt, dass der Satz, um wahr oder falsch sein zu können, erst sinnvoll sein muss ${ }^{23}$. Bestimmt man die Bedeutungen der undefinierbaren Symbole wie Namen und darüber hinaus die allgemeine Satzform, bestimmt man zugleich den Sinn eines jeden konstruierbaren Satzes, d.h. seine Wahrheitsbedingungen ${ }^{24}$. Nehmen wir an, es gäbe diese Forderung der Bestimmtheit des Sinnes des Satzes durch die Konventionen über Bedeutungen von Undefinierbaren und die allgemeine Satzkonstruktionsvorschrift nicht. Dass der Satz Sinn hat, wäre dann eine logische Voraussetzung dafür, dass der Satz wahr oder falsch sein kann. Um zu bestimmen, ob der Satz sinnvoll ist, d.h. um die Wahrheit oder Falschheit solcher Sätze wie „p ist ein sinnvoller Satz“ oder ,,p ist wahr oder falsch“ zu bestimmen, wäre die Definition der Wahrheitsbedingungen für solche Sätze im Symbolismus, nämlich bei seinem Aufbau ${ }^{25}$, erforderlich. Im Tractatus betrachtet Wittgenstein als Beispiel eines misslungenen Versuchs, eine solche Definition zu geben, die Russellsche Definition der Pro-

\footnotetext{
${ }^{19}$ Ebd., 2.21, 4.06

${ }^{20}$ Ebd., 3.144, 4.023

${ }^{21}$ Notes on Logic, 95

${ }^{22}$ TLP, 4.063

${ }^{23}$ Notes Dictated to Moore, 113

${ }^{24}$ Ebd., 112-113

${ }^{25}$ Als Beispiel einer solchen Definition kann man die Angabe der Bedingungen, unter welchen ein Name eine Bedeutung hat, in den Grundgesetzen der Arithmetik betrachten. S. GGA I, §§29, 30, 32, 33.
} 
position, die 1903 in The Principles of Mathematics ${ }^{26}$ gegeben wird. Dass eine solche Definition der Gestalt , $\mathrm{p} \supset \mathrm{p}$ “ dem Satz als Hypothese vorangestellt wird, garantiert nicht dafür, dass der Satz sinnvoll ist: Nicht die Hypothese, die im Fall, dass p kein Satz ist, unsinnig wird, macht den der Hypothese folgenden Satz unsinnig, sondern das Vorkommen in diesem des Nicht-Satzes selbst ${ }^{27}$.

Für den logischen Symbolismus bedeutet dieses Prinzip der Unterscheidung zwischen Sinn und Bedeutung und der Definierbarkeit des Sinnes eines Satzes durch die Bedingungen seiner Wahrheit, dass es verschiedene Kategorien von Symbolen gibt, die verschiedene Funktionen in bezug auf die Wirklichkeit, die ihnen zugeordnet wird, erfüllen: Einige Symbole (Sätze) können die Wirklichkeit in verschiedene Gebiete teilen („den Spielraum, der den Tatsachen“ von ihnen „gelassen wird“, beschränken ${ }^{28}$ ) und somit kraft ihrer symbolischen Eigenschaften ihre Beziehungen zu anderen Symbolen, z.B. Folgebeziehungen zu ihren Prämissen oder Folgen, bestimmen.

2. Zusammengesetzte Sätze, welche logische Konstanten enthalten, sagen nichts über Elementarsätze aus, deren Zeichen den Bestand der zusammengesetzten Satzzeichen ausmachen.

Das bedeutet, dass die logischen Konstanten „nicht vertreten“ und insbesondere keine Zeichen von Beziehungen $\operatorname{sind}^{29}$. So erörtert Wittgenstein in den Tagebüchern die Möglichkeit, dass ein Satz von den Eigenschaften eines Sachverhaltes oder eines anderen Satzes handeln könnte ${ }^{30}$. Zunächst nimmt er an, dass dies möglich wäre und dass ein solcher Satz tatsächlich eine zusätzliche materielle Information liefern könnte. Für den Satz „Es ist gut, dass p“ bedeutete dies, dass der Wahrheitswert dieses Satzes von einem anderen Faktor abhinge als nur von dem Wahrheitswert von p. Dieser Faktor könnte ein weiterer Satz sein, dessen Subjekt oder Argument entweder der Satz „p“ selbst oder sein Subjekt wäre, so dass man den Satz „Es ist gut, dass p“ als ein logisches Produkt des Satzes ,p“ und eines weite-

\footnotetext{
${ }^{26}$ Principles, §14

${ }^{27}$ TLP, 5.5351

${ }^{28}$ Ebd., 4.463, vgl. Notes on Logic, 102.

${ }^{29}$ TLP, 4.0312, 5.42, 5.461

${ }^{30}$ Tagebücher, 20.9.14
} 
ren Satzes darstellen könnte. Im ersten Fall, wenn das Argument des von $p$ verschiedenen Satzes der Satz ,p“ selbst wäre, könnte man den

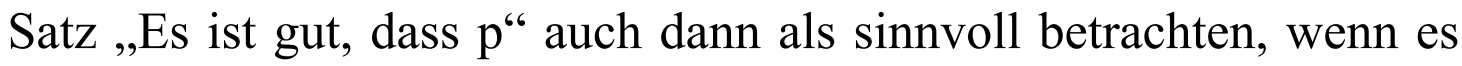
nicht der Fall wäre, dass p. Die Möglichkeit dieser Betrachtungsweise bedeutete, dass der Wahrheitswert des Produkts der beiden Faktoren von dem Wahrheitswert von $p$ unabhängig wäre, was laut Russell und Whitehead auf die sogenannten intensionalen propositionalen Funktionen und Propositionen wie $A$ glaubt, dass $p$ zutrifft ${ }^{31}$. Sofern $\mathrm{p}$ in dem fraglichen Satz beurteilt wird, kann man annehmen, dass

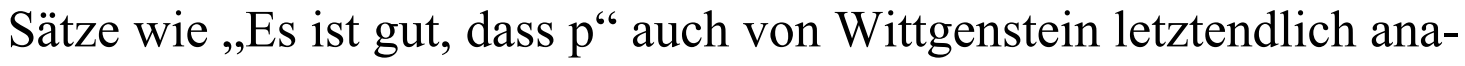
$\log$ den Sätzen wie „A glaubt, dass p“ gedeutet wurden. Im zweiten Fall, wenn das Argument des von p verschiedenen Satzes das Subjekt des Satzes ,„p“ wäre, würde jeder Faktor des Produkts etwas ein und demselben Subjekt, nämlich dem Subjekt von p, zuschreiben. Die Möglichkeit dieser Auffassung betrachtet Wittgenstein als Widerlegung der Unmöglichkeit der Analyse, die jeden Satz, der scheinbar etwas über die Eigenschaft eines anderen Satzes aussagt, als Wahrheitsfunktion von bestimmten Elementarsätzen darstellt.

Für zusammengesetzte Symbole fordert dieses Prinzip der Unaussprechbarkeit der logischen Eigenschaften des Satzes sowie der logischen Beziehungen zwischen Sätzen, das Symbol von seiner Zeichengestalt zu unterscheiden. Nicht jedes Zeichen, dass zum Zeichenbestand des Symbols gehört, erfüllt eine selbständige Funktion im Symbolismus und ist selbst ein Symbol.

3. Logische Konstanten sind deswegen keine Funktionen. Wahrheitsfunktionen sind Sätze: Zusammengesetzte Sätze sind Wahrheitsfunktionen von Elementarsätzen und jeder Elementarsatz „,ist eine Wahrheitsfunktion seiner selbst ${ }^{\text {‘32 }}$. Wahrheitsfunktionen sind von Wahrheitsoperationen $\mathrm{zu}$ unterscheiden, welche Elementarsätze als ihre Basen haben und Wahrheitsfunktionen als Resultate ihrer Anwendung ergeben. Die Wahrheitsoperation wird von Wittgenstein deswegen als die Art und Weise definiert, ,wie aus Elementarsätzen die Wahrheitsfunktion entsteht $t^{633}$. Eine Wahrheitsoperation kann auf ei-

\footnotetext{
${ }^{31} \mathrm{PM}, 72-73$

${ }^{32}$ TLP, 5

${ }^{33}$ Ebd., 5.3
} 
ne Wahrheitsfunktion sukzessiv angewandt werden, so dass das Resultat der Anwendung der Operation zu ihrer Basis werden kann und die Möglichkeit, dass die Wahrheitsfunktion als ihr eigenes Argument auftritt, ausgeschlossen wird ${ }^{34}$. Sind zwei Resultate der Anwendung von logischen Operationen auf Elementarsätze dieselbe Wahrheitsfunktion, sind diese Resultate identisch ${ }^{35}$.

Für den logischen Symbolismus bedeutet dieses Prinzip der Unterscheidung zwischen Wahrheitsfunktionen und Wahrheitsoperationen und der Abhängigkeit der Wahrheitsbedingungen eines Satzes von den Wahrheitsmöglichkeiten von Elementarsätzen zunächst, dass man ein Symbol von der es erzeugenden symbolischen Operation unterscheiden soll. Darüber hinaus kann durch die Anwendung verschiedener symbolischer Operationen ein und dasselbe Symbol erzeugt werden, was bedeutet, dass symbolische Operationen in bestimmten Beziehungen zueinander stehen und solche Beziehungen definierbar sind.

4. Symbole für Wahrheitsoperationen ${ }^{36}$ erfüllen die Funktion von Konstruktionsvorschriften, nach welchen Wahrheitsfunktionen gewonnen werden. Die Voraussetzung dafür, dass sie als Konstruktionsvorschriften fungieren können, besteht darin, dass es Elementarsätze gibt, die als Basen der Anwendung von Operationen dienen ${ }^{37}$ und die Vielfalt der Operationen bestimmen ${ }^{38}$. Die Existenz der Elementarsätze ist aus logischen Gründen gefordert ${ }^{39}$.

Folglich besteht die Voraussetzung des Aufbaus eines logischen Symbolismus in der Definition einer Menge von Grundsymbolen.

Der Bestand der Theorie des Zeigens lässt sich durch folgende Prinzipien definieren:

1. Es gibt etwas, das in dem Zeichen nicht zum Ausdruck kommt und das durch die Anwendung des Zeichens gezeigt wird ${ }^{40}$. Was in dem

\footnotetext{
${ }^{34}$ Ebd., 5.251, 5.2523, 5.253, 5.32

${ }^{35}$ Ebd., 5.41

${ }^{36}$ Ein Beispiel eines solchen Symbols ist die im Satz 6 des Tractatus definierte allgemeine Form des Satzes.

${ }^{37}$ TLP, 5.01, 5.442

${ }^{38}$ Vgl. ebd., 5.47.

${ }^{39}$ TLP, 5.555, 5.5562

${ }^{40}$ Ebd., 3.262
} 
Zeichen zum Ausdruck kommt, ist dagegen das, was einer in der Sprache ausdrückt oder was er durch den Satz sagt ${ }^{41}$. Was einer in der Sprache ausdrückt oder was der Satz darstellt, ist die Wirklichkeit (Tatsache), aber nicht die logische Form der Wirklichkeit, die der Wirklichkeit und der Sprache gemeinsam ist und die ,sich in der Sprache“" ausdrückt ${ }^{42}$. Dieses Prinzip der Unterscheidung zwischen dem Gesagten und Gezeigten kann man als die Forderung auffassen, das In-dem-Satz-Gesagte von dem Über-den-Satz-Gesagten zu trennen. Wittgenstein fordert diese Trennung, indem er die Möglichkeit des Sagens über den Satz ausschließt und die Funktion des Satzes, etwas über seine eigenen logischen Eigenschaften oder die logischen Eigenschaften eines anderen Satzes auszusagen, durch die Funktion des Satzes, seine eigenen logischen Eigenschaften sowie die logischen Eigenschaften seiner strukturellen Bestandteile zu zeigen, ersetzt. Durch diese Ersetzung wird gleichzeitig die Möglichkeit ausgeschlossen, den logischen Symbolismus oder seine Teile als eine Metasprache, d.h. die Sprache der Aussagen über eine gegebene natürliche oder formalisierte Sprache, zu betrachten.

Die Aufgabe des zusammengesetzten Symbols in einem anwendbaren logischen Symbolismus ist somit nicht auf eine mitteilende Funktion reduzierbar. Wenn die symbolisierende Funktion des Symbols in seiner Anwendung besteht ${ }^{43}$, kann es sogar Symbole außer sinnvollen Sätzen und Namen geben.

Die mitteilende Funktion des Symbols kann dennoch als Kriterium dienen, mit dessen Hilfe Symbole, die eine ,Verbindung mit der Welt" haben, von denjenigen Symbolen, die von nichts ,'handeln'“, unterschieden werden ${ }^{44}$. Diese Unterscheidung kann man, den früheren Arbeiten Wittgensteins folgend, als Unterscheidung der Symbolisierungsweise, d.h. dessen, „wie“ ein Symbol etwas zeigt, auffassen. So besteht die Art des Zeigens, welche die logischen Sätze von allen anderen Sätzen unterscheidet, darin, dass die logischen Sätze Tauto-

\footnotetext{
${ }^{41}$ Ebd., 4.062, 4.121

${ }^{42}$ Ebd., 4.121

${ }^{43}$ Vgl. ebd., 3.262.

${ }^{44}$ Vgl. ebd., 6.124.
} 
logien $\operatorname{sind}^{45}$. Dieser These folgend, könnte man die Art, wie die nicht-logischen Sätze etwas zeigen, darin sehen, dass sie Sinn haben $^{46}$, d.h. wahr oder falsch sein können ${ }^{47}$. Durch die Bestimmung der Symbolisierungsweise wird das Symbol in eine bestimmte Kategorie von Symbolen eingereiht. Diese Einreihung kann man als Analogon der semantischen Interpretation des Symbols betrachten ${ }^{48}$, von welcher man in jeder modernen Darlegung einer formalen logischen Theorie spricht. Diese Betrachtungsweise liegt auch deswegen nahe, weil die Regeln der semantischen Interpretation in der Logik für jede Kategorie von Symbolen gesondert aufgestellt werden.

2. Wenn der Satz die logische Form der Wirklichkeit zeigt, zeigt er sie durch seine Struktur ${ }^{49}$. Die Eigenschaften der Struktur und die Beziehungen von Strukturen bezeichnet Wittgenstein als formale oder interne Eigenschaften und Relationen ${ }^{50}$. Das Bestehen formaler Eigenschaften einer Sachlage drückt sich durch interne Eigenschaften des Satzes, der die Sachlage darstellt, aus, und das Bestehen einer formalen Relation zwischen möglichen Sachlagen drückt sich durch eine interne Relation zwischen den die Sachlagen darstellenden Sätzen aus ${ }^{51}$. Dabei kann man einem Satz seine formalen Eigenschaften oder formalen Relationen zu anderen Sätzen weder zusprechen noch absprechen $^{52}$.

Bereits in den „Notes dictated to G.E. Moore in Norway“ (1914), die fast ausschließlich der Unterscheidung zwischen Sagen und Zeigen gewidmet sind, unterscheidet Wittgenstein zwischen zusammengesetzten und nichtzusammengesetzten Symbolen, insbesondere Namen, und definiert für die ersteren, was sie zeigen. Wird eine Art der Symbole durch Definition oder „Beschreibung“ ausgezeichnet, und wird von Symbolen der ausgezeichneten Art festgestellt, dass man sie dadurch gewinnt, dass man gewisse andere Symbole der Sprache

\footnotetext{
${ }^{45}$ Notes Dictated to Moore, 114

${ }^{46}$ Ebd., 108

${ }^{47}$ Vgl. ebd., 113.

${ }^{48}$ Ich werde auf die Möglichkeit dieser Betrachtungsweise im weiteren zurückgreifen.

${ }^{49}$ TLP , 4.121, 4.1211

${ }^{50}$ Ebd., 4.122

${ }^{51}$ Ebd., 4.124, 4.125

52 Ebd., 4.124
} 
nach bestimmten Vorschriften kombiniert, dann zeigt eine solche Kombinierbarkeit irgendeine Eigenschaft der kombinierbaren Symbole $^{53}$. Die Rolle der ausgezeichneten Symbole erfüllen in einem logischen Symbolismus vor allem Tautologien, welche folglich die logischen Eigenschaften der Sprache zeigen. Die von ihnen gezeigten Eigenschaften sind Eigenschaften der Sätze, welche Teilsätze der tautologischen Sätze bilden.

Mithin verdanken Symbole ihre symbolisierende Funktion ihrer Struktur. Der strukturelle Charakter eines Symbols ermöglicht seine Zuordnung zu Elementen und Merkmalen der strukturierten Wirklichkeit und so bedingt auch die Anwendung des logischen Symbolismus.

3. Strukturen von Sätzen stehen in internen Relationen zueinander. Solche Relationen können ,hervorgehoben“ werden, wenn man einen Satz als Resultat einer Operation auf einem anderen als Basis der Operation auftretenden Satz darstellt. Die Operation bringt den Unterschied der Formen ihrer Basen und Resultate zum Ausdruck ${ }^{54}$.

Die Transformation des Symbols in einem logischen Symbolismus durch die Anwendung symbolischer Operationen ist folglich durch den strukturellen Charakter des Symbols bedingt.

4. Ein Satz kann laut Wittgenstein in einem anderen Satz nur als Basis einer Wahrheitsoperation vorkommen ${ }^{55}$.

Diese These Wittgensteins ist zunächst der Ausdruck der Idee, dass Sätze keine Namen sind. 1914 äußert Wittgenstein diese Idee in Form der Forderung, eine interne Beziehung zwischen Sätzen von der internen Beziehung zwischen Satz und Namen zu trennen, wobei er die letztere Beziehung als das Vorkommen des Namens in einem Satz charakterisiert ${ }^{56}$.

Im Symbolismus müssen zusammengesetzte Symbole nach einer Regel konstruiert werden: Die Aufgabe einer solchen Regel besteht darin, die Anwendung symbolischer Operationen auf die Symbole zu regeln, die als Basen von Operationen per Definition eingeführt wer-

\footnotetext{
${ }^{53}$ Notes Dictated to Moore, 108

${ }^{54}$ TLP, 5.2, 5.21, 5.22, 5.24

55 Ebd., 5.54

${ }^{56}$ Notes Dictated to Moore, 111, 116
} 
den und die Grenze der durch die Regel vorgesehenen strukturellen Analyse der Symbole bilden.

5. An einem Symbol unterscheidet Wittgenstein verschiedene Züge. So besitzt ein Satz zufällige, von der besonderen Art der Hervorbringung des Satzes herrührende, und wesentliche, den Satz zum Ausdrücken seines Sinnes befähigende, Züge ${ }^{57}$. Charakteristische Züge des Symbols sind laut Wittgenstein Ausdrücke seiner formalen Eigenschaften $^{58}$. Das Vorkommen eines Zeichens in einem Symbol, z.B. des Verneinungszeichens in einem Satz, ist nur dann wesentlich, wenn es den Sinn des Satzes charakterisiert ${ }^{59}$. Diese These ist die Entwicklung des in den „Notes on Logic“ geäußerten Gedankens, dass das, was in zwei molekularen Wahrheitsfunktionen mit demselben Wahrheitsschema symbolisiert, dasselbe ist ${ }^{60}$.

Dass nicht jeder Zug des Symbols symbolisiert, bedeutet für den Symbolismus, dass sich das Symbol von einem Zeichengebilde unterscheidet und dass dieser Unterschied einer Festlegung durch bestimmte Konventionen des Symbolismus (durch seine Regeln) bedarf.

6. Einen Zug des Symbols, der seine formalen Eigenschaften ausdrückt, kann man in der Konstanz erkennen, die eine Satzvariable aufweist ${ }^{61}$. Jede Satzvariable bezeichnet einen formalen Begriff, dessen Merkmale formale Eigenschaften der Symbole sind, deren Bedeutungen unter den formalen Begriff fallen ${ }^{62}$. So ist der variable Name , $\mathrm{X}_{\text {“ }}$ für Wittgenstein das Zeichen des formalen Begriffs „Gegenstand“. Dieser Name kann auf dieselbe Weise in verschiedenen variablen Sätzen vorkommen. Zu Eigenschaften eines jeden Namens gehört deshalb, dass der Name eines Gegenstandes auf dieselbe Weise in verschiedenen Symbolen, nämlich Sätzen, vorkommen kann ${ }^{63}$. Formale Eigenschaften eines Symbols charakterisieren es durch seine Bezie-

\footnotetext{
${ }^{57}$ TLP, 3.34

${ }^{58}$ Ebd., 4.126

${ }^{59}$ Ebd., 4.0621

${ }^{60}$ Notes on Logic, 99

${ }^{61}$ TLP, 4.126

${ }^{62}$ Ebd., 4.126, 4.127, 4.1272

${ }^{63} \mathrm{Vgl}$. Notes on Logic, 96.
} 
hungen zu anderen Symbolen und kennzeichnen somit das Symbol als eines, das zu einer bestimmten Kategorie gehört ${ }^{64}$.

Verschiedene Kategorien von Symbolen eines logischen Symbolismus sind mithin durch ihre gegenseitigen Beziehungen charakterisiert.

7. Dass nicht jeder Zug des Symbols symbolisiert, bedeutet für Wittgenstein, dass das Symbolisierende am Symbol einer Definition bedarf. Das Symbolisierende am Symbol sieht Wittgenstein in dem Gemeinsamen der Symbole, die denselben Zweck erfüllen und deswegen für einander substituierbar sind ${ }^{65}$.

Es kann folglich verschiedene Symbolismen geben, die demselben Zweck dienen.

Zwischen Prinzipien und Thesen der drei Theorien besteht ein Zusammenhang. So kann man die Forderung, zwischen Sagen und Zeigen zu unterscheiden, als Ergebnis der Gleichsetzung des Sinnes des Satzes mit seinen Wahrheitsbedingungen und Zurückführung der Wahrheitsbedingungen eines sinnvollen Satzes auf die Wahrheitsbedingungen von Elementarsätzen, die seine Wahrheitsargumente sind, ansehen. Durch diese von der Theorie der Wahrheitsfunktionen verlangte Zurückführung wird die Abhängigkeit des Sinnvoll-seins eines Satzes von dem Wahrheitswert einer Aussage über den Satz und folglich die Möglichkeit, dem Satz etwas zu prädizieren, ausgeschlossen. Ihrerseits impliziert die Forderung, zwischen Sagen und Zeigen zu unterscheiden, zusammen mit der Behauptung, dass es Sätze, nämlich Tautologien, gibt, die nichts sagen, dafür aber etwas zeigen, die These der Theorie der Wahrheitsfunktionen, laut welcher die logischen Konstanten wie Verneinungs- oder Disjunktionszeichen keine Namen für Beziehungen zwischen Sätzen sind und deswegen nicht vertreten ${ }^{66}$ : Könnten sie dies tun, wären Tautologien, die aus sinnvollen Sätzen, die durch logische Konstanten verknüpft sind, bestehen, auch etwas sagen.

Obwohl ein derartiger Zusammenhang von Prinzipien der oben definierten Theorien nicht jedem Zusammenhang von Prinzipien, die für Symbole ei-

\footnotetext{
${ }^{64} \mathrm{Vgl}$. Notes Dictated to Moore, 116.

${ }^{65}$ TLP, 3.341, 3.344, vgl. Notes Dictated to Moore, 117.

${ }^{66}$ Notes on Logic, 101, Notes Dictated to Moore, 116, TLP, 4.0312
} 
nes logischen Symbolismus formuliert werden können, entspricht ${ }^{67}$, lässt sich aus den unten formulierten Prinzipien für Symbole der Zusammenhang der drei Theorien erkennen. Als Ausdruck dieses Zusammenhangs kann man die Charakterisierung des logischen Symbolismus betrachten, die durch die Prinzipien für Symbole gegeben ist. Diese Charakterisierung ist folgende:

1. Das Symbol unterscheidet sich von einem Zeichengebilde. Ein Zeichengebilde ist nur dann ein Symbol, wenn es bestimmte Bedingungen erfüllt. Insbesondere ist nicht jedes Zeichen, das ein Bestandteil eines Symbols ist, ein selbständiges Symbol, das ohne Zusammenhang mit anderen Zeichen und Symbolen gebraucht werden kann.

2. Das Symbol hat eine Struktur: Dank seiner Struktur ist das Symbol mit anderen Symbolen verbunden. Die Verbindung des Symbols mit anderen Symbolen realisiert sich in der Transformierbarkeit des Symbols. Dass das Symbol eine Struktur hat, ist darüber hinaus Voraussetzung der Anwendbarkeit des Symbolismus, sofern seine Anwendung auf den Zuordnungen der Elemente des Symbols zu Elementen der Wirklichkeit beruht und darauf gründet, dass die Zuordnung die strukturellen Beziehungen zwischen den Elementen des Symbols einerseits und den Elementen der dem Symbol zugeordneten Wirklichkeit andererseits erhält.

3. Die Transformationen der Symbole im Symbolismus, d.h. die Anwendungen der aus gegebenen oder bereits gewonnenen Symbolen neue Symbole erzeugenden Operationen, unterliegen bestimmten Regeln. Die Transformationen dienen zunächst der Konstruktion der Symbole. Diese Konstruktion ist geregelt und beruht auf bestimmten Voraussetzungen. Die Voraussetzung des Aufbaus eines logischen Symbolismus ist eine Definition seiner Grundsymbole. Die Regeln, die dem Aufbau des Symbolismus zugrunde liegen, haben zwar den syntaktischen Charakter ${ }^{68}$, berücksichtigen jedoch die Anwendung des Symbolismus, sofern sie auf Zuordnungen der Elemente des Symbols zu Elementen der Wirklichkeit gründen. Die konstruktiven

\footnotetext{
${ }^{67}$ Die Erklärung für das fehlende Entsprechen besteht darin, dass die Formulierung der Prinzipien für Symbole keine direkte Übersetzung der Prinzipien der definierten Theorien ist, sondern eher Folgerungen aus den letzteren Prinzipien beinhaltet.

${ }^{68} \mathrm{Vgl}$. TLP, 3.33.
} 
Regeln des logischen Symbolismus definieren somit einerseits die Arten von interpretierbaren Grundsymbolen, aus welchen alle anderen auf eine bestimmte Weise interpretierbaren Symbole des Symbolismus konstruiert werden ${ }^{69}$, und andererseits die konstruktiven Operationen des Symbolismus. Zusammen mit der Darstellung von zusammengesetzten Grundsymbolen des logischen Symbolismus (von Elementarsätzen) als Zeichen, die Wahrheitswerte-Abzeichen mitführen, sowie mit der Definition der logischen Sätze als Tautologien bedeutet die geregelte Konstruktion der Symbole im logischen Symbolismus, dass an ihn die Forderung der syntaktischen Lösung des Problems der Entscheidbarkeit gestellt wird. Diese Forderung formuliert Wittgenstein in Form der Behauptung darüber, dass es in der Logik keine Überraschungen geben $\operatorname{kann}^{70}$.

4. Dass im logischen Symbolismus Tautologien konstruierbar sind, bedeutet unter anderem, dass es in einem anwendbaren logischen Symbolismus verschiedene Kategorien von Symbolen gibt. Solche Kategorien unterscheiden sich von einander zunächst durch ihre Beziehung zur Wirklichkeit, die sich in der Unterscheidung ihrer Funktionen in bezug auf die Wirklichkeit äußert. Verschiedene Kategorien von Symbolen kann man durch ihre gegenseitigen Beziehungen charakterisieren, was den logischen Symbolismus zu einem strukturierten Ganzen macht. Insbesondere kann man Symbole von einander durch den Beitrag unterscheiden, welchen sie zur Strukturierung des Symbolismus leisten: Im logischen Symbolismus ist dieser Beitrag vor allem dadurch bestimmt, ob Symbole als Ausdrücke von Wahrheitsbedingungen interpretiert werden können und ob solche Bedingungen für das Symbol tautologisch, kontradiktorisch oder weder tautologisch noch kontradiktorisch sind ${ }^{71}$. Die Wahrheitsbedingungen des Symbols Satz zusammen mit seinen Wahrheitsargumenten

${ }^{69}$ Die konstruktive Regel, die Wittgenstein formuliert, behandelt als Grundsymbole Elementarsätze, so dass die Interpretationsweise der Grundsymbole und der im Symbolismus konstruierbaren Symbole dieselbe ist.

${ }^{70} \mathrm{Vgl}$. TLP, 6.124-6.1251, 6.126-6.1261.

${ }^{71}$ Vgl. ebd., 4.431, 4.46-4.461. 
bestimmen nach Wittgenstein die Stelle des Satzes im Symbolismus, weil sie die Beziehungen des Satzes zu anderen Sätzen bestimmen ${ }^{72}$.

5. Es kann verschiedene logische Symbolismen geben, die denselben Zweck erfüllen. Diese Möglichkeit beruht auf dem „Anteil“ des Willkürlichen, der die Konstruktion des Symbolismus bestimmt und der sich z.B. in verschiedenen Zuordnungen, die dem Symbolismus zugrunde liegen $^{73}$, oder in der Wahl der konstruktiven Operationen des Symbolismus ${ }^{74}$ äußert. Das Vorhandensein dieses Anteils schließt nicht aus den nicht-willkürlichen Charakter des Aufbaus des Symbolismus nach festgelegten Regeln sowie seiner Anwendung ${ }^{75}$.

${ }^{72}$ Vgl. ebd., 3.41, 4.0641.

${ }^{73}$ Vgl. ebd., 5.473.

${ }^{74}$ Vgl. ebd., 3.3441.

${ }^{75}$ Vgl. ebd., 3.342, 3.3442, 5.473. 
